\title{
Emergent Lorentz invariance in fermion sector
}

\author{
Ivan Kharuk ${ }^{1,2, \star}$ \\ ${ }^{1}$ Institute for Nuclear Research of the Russian Academy of Sciences, 60th October Anniversary Prospect, \\ 7a, 117312 Moscow, Russia \\ ${ }^{2}$ Moscow Institute of Physics and Technology, Institutskii per. 9, Dolgoprudny 141700, Moscow Region, \\ Russia
}

\begin{abstract}
By using holographic description of strongly interacting field theories we show that under common assumptions Lorentz invariance emerges as an effective low-energy symmetry of the theory, despite fundamental theory at hight energies being Lorentzviolating. We consider fermions sector and show that the notion of chirality also automatically arises in the infrared.
\end{abstract}

\section{Introduction}

The idea that Lorentz-invariance (LI) can be not a fundamental symmetry of nature but accidental or emergent symmetry of low-energy physics has been subject of studies for a while. In condensed matter physics such scenarios are rather common [1-3]: if one takes non-relativistic system and examines its renormalization group (RG) flow towards infrared (IR), then, typically, the effective Lagrangian would be LI, up to some suppressed corrections. This observation provides motivation for studying analogous models in particle physics, thus allowing to abandon LI at high energies. The last idea is interesting in context of models of modified gravity, particularly in case of Hórava - Lifshitz gravity [4], as such assumptions allow to improve ultraviolet (UV) behaviour of gravity and may help to explain some phenomena in cosmology.

The pioneering work in that direction in particle physics were made by Nielsen, Chadha and Ninomiya [5, 6], who studied quantum electrodynamics and Yang-Mills theory with Lorentz-violating (LV) coupling of dimension four and showed that they vanish in the IR limit of RG flow. However, their model fails to satisfy the experimental constrains, coming from precision test of LI [7, 8] - as in any weakly-coupled system, the marginal LV operators have logarithmic dependence on RG scale, that is not enough to stay within existing bounds on LV corrections. Indeed, if such corrections would be of order one at some energy scale $\Lambda_{*}$ (smaller or equal to Plank scale), then they would be only mildly suppressed at experimentally accessible energies.

There are two ways to overcome that problem. The first one is to impose additional symmetries on the model, that would forbid marginal LV couplings. In this case, the resulting theory in the IR would automatically be LI, as would contain only irrelevant LV operators. The other way is to turn to strong coupling regime, that may change the dependence of marginal coupling on RG scale from logarithmic to power-law one, that allows to avoid contradictions with experimental constrains. The second option

^e-mail: ivan.kharuk@phystech.edu 
is the one we are interested in, and we will show, by using gauge-gravity duality to describe strongly coupled system, that LV corrections do become power-law suppressed. What is more, we also show that the notion of chirality emerges together with LI, thus solving the puzzle how Weyl fermions one of the irreducible representations of Lorentz group - can appear in non-relativistic system.

The scenario we will be considering is the following. At energy higher than $\Lambda_{*}$ the theory is governed by some fundamental description, that does not include LI as a symmetry. Then, at energies of order $\Lambda_{*}$, the theory becomes strongly coupled and passes through strong RG evolution down to energy scale $\Lambda_{I R}$, at which it enters a confining phase. This implies that the Standard Model particles are composite ones. This property should become observable at energies of order $\Lambda_{I R}$, so that it should be bigger than experimentally accessible energies, as no such signal has been observed yet. We will be considering fermion fields, see also [9] for a more complete discussion on the that model for boson fields.

The paper is organised as follows. In the first section we consider a non-relativistic fermion coupled to a relativistic strongly interacting sector. In the second section we consider a more general set-up, describing RG flow from UV theory with anisotropic Lifshitz scaling down to LI IR fixed point. Finally, we conclude and summarize Sec. (4).

\section{Chiral LI fermions from non-relativistic systems}

\subsection{Holography for fermions}

The holographic construction for fermions is presented in [10,11], here we will use some of its results and adopt the model for our purposes. From here and in what follows capital Latin letters from the beginning of the alphabet are used as indices in local Lorentz frame, from the middle - for $(\mathrm{d}+1)-$ dimensional indices in AdS. Greek letters are used for flat directions, $t$ and $x^{i}$, where small Latin letters are used for purely spatial directions. In Poincare patch AdS metric has the form

$$
g_{M N}=\left(\frac{l}{u}\right)^{2} \eta_{\mu \nu}, \quad i=1, \ldots, d-1,
$$

where $\eta_{\mu v}=(-,+, \ldots,+)$ is flat mostly plus metric and $l$ is AdS radius. For simplicity, we will assume $(d+1)$ to be even, thought all of the results can be generalized to odd dimensionality of space-time.

In order to make possible introduction of fundamental LV fermion and to introduce confinement into the theory, we set two branes in the AdS, one in the UV and one in the IR, located at $\epsilon$ and $L$ respectively. To proceed one needs to solve for Dirac equation, coming from the standard action

$$
S=-\int d^{d+1} x \sqrt{-g} i(\bar{\psi} \not D \psi-M \bar{\psi} \psi)+S_{\delta},
$$

where all quantities are written in local Lorentz frame and $S_{\delta}$,

$$
S_{\delta}=-i \int_{u=\epsilon} d^{d} x(\epsilon / l)^{-\frac{d-1}{2}} \bar{\psi}_{-} \psi_{+},
$$

is introduced to cancel the term, coming from integration by parts after varying (2) with respect to $\psi$. The important step step to be done is to decompose $\psi$ into $\psi_{+}$and $\psi_{-}$, that are eigenvectors of $\Gamma^{u}$, the analogy of $\gamma^{5}$ in four dimensions:

$$
\psi=\psi_{+}+\psi_{-}, \quad \Gamma^{u} \psi_{ \pm}= \pm \psi_{ \pm} .
$$


That allows to solve the Dirac equation,

$$
\begin{aligned}
& \psi_{-}(\vec{p}, u)=(p u)^{(d+1) / 2}\left(\chi_{1}(\vec{p}) J_{M l+1 / 2}(p u)+\chi_{2}(\vec{p}) Y_{M l+1 / 2}(p u)\right), \\
& \psi_{+}(\vec{p}, u)=(p u)^{(d+1) / 2} \frac{i p_{\mu} \Gamma^{\mu}}{p}\left(\chi_{1}(\vec{p}) J_{M l-1 / 2}(p u)+\chi_{2}(\vec{p}) Y_{M l-1 / 2}(p u)\right),
\end{aligned}
$$

where $p=\sqrt{-p_{\mu} p^{\mu}}$. Depending on the value of $M l$, one or the other of the components is leading in the $u \rightarrow 0$ limit. We will focus on the case $M l \geq-\frac{1}{2}$, when $\psi_{-}$acts as a source for dual theory, the opposite choice is equivalent to that one up to some trivial substitutions. In this case on the CFT side we have dual fermion operator of dimension

$$
\operatorname{dim} O_{\psi}=M l+\frac{d}{2},
$$

that can be read-out from the behaviour of $\psi_{-}$near UV boundary. Note also that in the regime $-\frac{1}{2} \leq M l \leq \frac{1}{2}$ the dual CFT theory admits two alternative quantizations.

There are two possible boundary conditions (BC) on the IR brane, that we well denote as $\mathcal{B}_{+}$and $\mathcal{B}_{-}$:

$$
\begin{array}{lll}
\mathcal{B}_{+}: & \left.\psi_{-}\right|_{L}=0, & \left.\psi_{+}\right|_{L}-\text { arbitrary } \\
\mathcal{B}_{-}: & \left.\psi_{+}\right|_{L}=0, & \left.\psi_{-}\right|_{L}-\text { arbitrary }
\end{array}
$$

The spectrum of the theory depends on which BC are imposed. Direct calculations show that there are no massless modes in $\mathcal{B}_{+}$case, while in $\mathcal{B}_{-}$there is one of the form

$$
\psi_{+}=\chi_{0}\left(\frac{u}{l}\right)^{M l+\frac{d}{2}}, \quad \psi_{-}=0
$$

As it was explained in the introduction, in this set-up we would like to introduce fundamental massless LV fermion. Of course, this changes the resulting spectrum - in $\mathcal{B}_{+}$case there would be a gapless mode, coming from LV fermion, while in the $\mathcal{B}_{-}$case, as it can be directly checked, there would be no massless excitations. As our primary goal is to study emergence of chiral fermions, and as they can emerge only from the gapless mode, in this section we will consider $\mathcal{B}_{+}$boundary conditions.

\subsection{Emergence of LI chiral fermions}

Lets now introduce LV fermion. In AdS this is done by adding LV action for $\psi_{-}$on the UV brane. For simplicity, we take its action to be

$$
S_{U V}=-b \int d^{d} x i\left(\bar{\psi}_{-} \gamma^{0} \partial_{0} \psi_{-}+v \bar{\psi}_{-} \gamma^{i} \partial_{i} \psi_{-}\right)
$$

where parameter $v \neq 1$ explicitly breaks LI and $b$ is some parameter (of dimension length), introduced to make action dimensionless.

From the CFT point of view it is equivalent to promoting the source $\chi$ to a dynamical field at UV and introducing the interaction between the initial CFT and $\chi$,

$$
S=S_{C F T}+S_{\chi}+\Lambda_{i n t} \int d^{d} x \bar{\chi} O_{\psi}
$$


where $S_{\chi}$ is LV action for $\chi$,

$$
S_{\chi}=-b \int d^{d} x i\left(\bar{\chi} \gamma^{0} \partial_{0} \chi+v \bar{\chi} \gamma^{i} \partial_{i} \chi\right)
$$

that directly translates to (9).

The dimension of elementary fermion field $\chi$ is $\frac{d-1}{2}$, and the dimension of $O_{\psi}$ is given by (6). That allows to find the dimension of the coupling constant $\Lambda_{\text {int }}$, that turns out to be relevant only in the range

$$
-\frac{1}{2} \leq M L \leq \frac{1}{2}
$$

If $M l \geq \frac{1}{2}$, the coupling is irrelevant, so that one expects those two sectors to decouple in the IR and, hence, there is no reason for LI to emerge at low-energy limit. Direct calculations show that this is indeed the case in that regime. Thus, further we will focus only on the regime (12).

The boundary condition on the UV brane then reads

$$
\left.\left[b\left(\gamma^{0} \partial_{0}+v \gamma^{i} \partial_{i}\right) \psi_{-}+\psi_{+}\right]\right|_{u=l}=0 .
$$

Together with IR boundary condition that leads the solution [11]

$$
\psi_{-}=\chi_{1} f_{-}(u), \quad \psi_{+}=\frac{i p_{\mu} \gamma^{\mu}}{p} \chi_{1} f_{+}(u),
$$

where

$$
f_{ \pm}(u)=(p u)^{(d+1) / 2}\left[J_{M l \mp 1 / 2}(p u)-\frac{J_{M l-1 / 2}(p L)}{Y_{M l-1 / 2}(p L)} Y_{M l \mp 1 / 2}(p u)\right],
$$

where $J_{\alpha}$ and $Y_{\alpha}$ are first and second kind Bessel functions respectively.

Our main interest is to study the low-energy behaviour of that theory. Assuming $p l \ll p L \ll 1$ and expanding the solution in Tylor series, we get the dispersion relation

$$
\omega= \pm|\mathbf{k}|\left[1+(v-1)(1-2 M l) \frac{b}{l}\left(\frac{l}{L}\right)^{1-2 M l}\right],
$$

where $\omega=-p_{0}, k_{i}=p_{i}$ and $\mathbf{k}=\sqrt{k_{i} k^{i}}$. It is LI up to corrections, that are power-law suppressed, as it was advertised in the introduction. If the ration $\frac{l}{L}$ is small enough, than this model passes all experimental constrains. What is more, more careful analysis also shows that it has exactly $2^{\frac{d}{2}-2}$ components (others being suppressed by the same factor), that coincides with the structure and number of components of Weyl fermion.

This also have a very clear interpretation on the CFT side. As it was mentioned earlier, in regime (12) dual theory admits two alternative quantizations, one with dimension of dual operator given by (6), and one with

$$
\operatorname{dim} O_{\psi}^{\prime}=-M l+\frac{d}{2} .
$$

The LV corrections to dispersion relation are reproduced by deforming the initial CFT by

$$
\delta S_{C F T} \sim \int d^{d} x \bar{O}_{\psi}^{\prime} \gamma^{0} \partial_{0} O_{\psi}^{\prime}
$$

Thus, the above model also describes RG flow between two alternative quantizations of bulk theory, that helps to restore LI in IR. 


\section{Fermions in Lifshitz flow}

\subsection{Geometry of Lifshitz flow}

The other possibility to consider is the case when fundamental theory at high energies has anisotropic isometries, that is of interest, for example, in context of Hórava gravity. This is more general set-up for studying non-relativistic field theories as there is no grantee a priori that time and space are similar in any sense in some fundamental theory. By definition, Lifshitz space, introduced in [12, 13], has anisotropic isometries

$$
t \mapsto \lambda^{z} t, \quad x_{i} \mapsto \lambda x_{i}, \quad u \mapsto \lambda u,
$$

By extrapolation of the logic of AdS/CFT duality, such spaces are claimed to be dual to "anisotropic CFT" and are expected to catch the strong coupling dynamics of such theories.

In order to make contact with phenomenology, one should construct a domain-wall geometry, that would interpolate between Lifshitz space at $u \rightarrow 0$ and AdS at $u \rightarrow \infty$. Such models were constructed and studied in [14], and here we will provide a very brief overview of its properties.

The required geometry can be obtained as a solution of Einstein equations with negative cosmological constant and a vector field with mass $M_{V}$. Provided that inequality

$$
d-1 \leq\left(M_{V} l\right)^{2} \leq \frac{d(d-1)^{2}}{3 d-4}
$$

is satisfied, LI fixed point in the infrared acts as attractor for RG flows, thus providing required geometry. From the dual viewpoint, this is equivalent to adding an operator of dimension

$$
\operatorname{dim} O_{V}^{\mu}=d+\alpha_{V}, \quad \alpha_{V}=-\frac{d}{2}+\sqrt{\left(\frac{d}{2}-1\right)^{2}+\left(M_{V} l\right)^{2}}
$$

that is irrelevant as long as $\alpha_{V}<0$, that can be checked to coincide with (20). In this set-up we are interested in the spectrum of pure CFT, without any sources, so that, in contrast to previous section, we would have no UV brane and impose $\mathcal{B}_{-}$boundary conditions on IR brane.

\subsection{Emergence of LI fermions in Lifshitz flows}

We would like to study the behaviour of fermions on such domain-wall background. The action of the model we are considering is

$$
\left.S=-\int d^{d+1} x \sqrt{-g} i \bar{\psi}(\not D-\xi \not D)^{(V)}-M\right) \psi
$$

where $\not D$ is a Dirac operator and $\not D^{(V)}$ introduces interaction between fermion and vector field,

$$
\not D^{(V)} \equiv \frac{\left(M_{V} l\right)^{2}}{4} V_{N} V_{M} e_{A}^{M} \Gamma^{A} D^{N} .
$$

The coupling constant $\xi$ can depend on the species of the fermion (if there were several of them, as it is required in realistic models), thus allowing to trace possible dependence of LV corrections on that coupling.

Avoiding technical details, the proof that there is a gapless chiral LI fermion in the IR goes as follows. First, one calculates the explicit view of Dirac operator and coupling $D^{(V)}$. Then the field $\psi$ 
should be again split into $\psi_{+}$and $\psi_{-}$, the eigenvalues of $\Gamma^{u}$, as in previous section. At this stage it is possible to set $\omega=k_{i}=0$ and show that there is a solution of a form

$$
\psi_{+}=\psi_{+}^{(0)}, \quad \psi_{-}=0
$$

where the exact expression for $\psi_{+}^{(0)}$ is unimportant for our purposes. The normalization integral for that mode is saturated in AdS region, that is, in the IR. But this means that we can consider the above solution as zero-th order approximation to the exact solution in some vicinity of $\omega=k_{i}=0$ and use perturbation series technique to calculate higher corrections. Implementing that idea in first order perturbations yields dispersion relation

$$
\omega^{(1)}=|\mathbf{k}|\left[a_{1}(1+2 M l)\left(\frac{l_{*}}{L}\right)^{2 \alpha_{V}}+a_{2}(1+2 M l)\left(\frac{l_{*}}{L}\right)^{1+2 M l}\right],
$$

with some coefficients $a_{1}$ and $a_{2}$ being functions of background metric and coupling constant $\xi$. Clearly, as long as $\alpha_{V}>0$ and $M l>-\frac{1}{2}$, LV correction are suppressed, so that LI do emerges in that regime, that matches nicely with the results, obtained in previous section. What is more, it can be shown that the corresponding mode is chiral.

Again, this results have clear interpretation on the CFT side. The resulting RG flow describes the evolution of CFT, deformed by

$$
\delta S_{C F T}=\int d^{d} x\left(c_{1} l_{*}^{\alpha_{V}} O_{V}^{0}+c_{2} l_{*}^{1+2 M l} \bar{O}_{\psi} \gamma^{0} \partial_{0} O_{\psi}\right)
$$

where $c_{1,2}$ are some dimensionless constants. They generate exactly the same corrections that appear in $(25)^{1}$.

\section{Conclusions}

Considering LV models in particle physics, we demonstrated that turning to strong coupling regime allows to overcome the problems, present in a weakly coupled scenarios. Namely, LV corrections become power-law suppressed, that allows to avoid contradictions with experimental data, provided that the hierarchy between $\Lambda_{*}$ and $\Lambda_{I R}$ is large enough. This means that the scenario of [9] is also applicable in fermion sector. What is more, the emergence of LI in this case is followed by the emergence of the notion of chirality, that is crucial for realistic theories, as Weyl fermion play central role in the Standard Model.

We observed that the exponent of suppression is bounded from above by 2 . Whether it is a fundamental constrain or not is left as an opened question. It is important to study this question as if there are theories with exponents grater than 2 it would be much harder to close them experimentally. Also, it would be interesting to include gauge fields into consideration, but this is left for future work, as such models would require more complicated bulk sector.

To summarize, our calculations show that fundamental theory can be not just LV, but also anisotropic one, still having a well-defined IR limit, with LI as an effective low-energy symmetry, having no contradictions with experimental bounds on LV effects.

\footnotetext{
${ }^{1}$ Only double insertion of operator $O_{V}^{0}$ modifies the dispersion relation due to presence of bulk symmetry $V_{M} \mapsto-V_{M}$ for a bulk vector field.
} 


\section{References}

[1] O. Vafek, Z. Tesanovic, M. Franz, Phys. Rev. Lett. 89, 157003 (2002) [cond-mat/0203047].

[2] D. J. Lee, I. F. Herbut, Phys. Rev. B 66, 094512 (2002) [cond-mat/0201088].

[3] I. F. Herbut, V. Juricic, B. Roy, Phys. Rev. B 79, 085116 (2009) [arXiv:0811.0610 [cond-mat.strel]].

[4] P. Horava, Phys. Rev. D 79, 084008 (2009) [arXiv:0901.3775 [hep-th]].

[5] H. B. Nielsen, M. Ninomiya, Nucl. Phys. B 141, 153 (1978).

[6] S. Chadha, H. B. Nielsen, Nucl. Phys. B 217, 125 (1983).

[7] D. Mattingly, Living Rev. Rel. 8, 5 (2005) [gr-qc/0502097].

[8] V. A. Kostelecky, N. Russell, Rev. Mod. Phys. 83, 11 (2011) [arXiv:0801.0287 [hep-ph]].

[9] G. Bednik, O. Pujolàs and S. Sibiryakov, JHEP 1311, 064 (2013) [arXiv:1305.0011 [hep-th]].

[10] M. Henningson and K. Sfetsos, Phys. Lett. B 431, 63 (1998) [hep-th/9803251].

[11] R. Contino, A. Pomarol, JHEP 0411, 058 (2004) [arXiv:hep-th/0406257].

[12] S. Kachru, X. Liu and M. Mulligan, Phys. Rev. D 78, 106005 (2008) [arXiv:0808.1725[hep-th]].

[13] M. Taylor, arXiv:0812.0530 [hep-th].

[14] H. Braviner, R. Gregory and S. F. Ross, Class. Quant. Grav. 28, 225028 (2011) [arXiv:1108.3067 [hep-th]]. 